\title{
Fast transition between Classical and Weak lined T Tauri stars due to external UV dissipation
}

\author{
Jin Zeng $\mathrm{Li}^{1}$, Travis A. Rector ${ }^{2}$
}

\begin{abstract}
The discovery of optical jets immersed in the strong UV radiation field of the Rosette Nebula sheds new light on, but meanwhile poses challenges to, the study of externally irradiated jets. The jet systems in the Rosette are found to have a high state of ionization and show unique features. In this paper, we investigate the evolutionary status of the jet driving sources for young solar-like stars. To our surprise, these jet sources indicate unexpected near infrared properties with no excess emission. They are bathed in harsh external UV radiation such that evaporation leads to a fast dissipation of their circumstellar material. This could represent a transient phase of evolution of young solar-like stars between classical and weak lined T Tauri stars. Naked T Tauri stars formed in this way have indistinguishable evolutionary ages from those of classical T Tauri stars resulting from the same episode of star formation. However, it would be hard for such sources to be identified if they are not driving an irradiated jet in a photoionized medium.
\end{abstract}

Subject headings: Stars: formation — Stars: pre-main sequence — Herbig-Haro objects - ISM: jets and outflows

\section{Introduction}

One of the major advancements in recent years in the study of Herbig-Haro (HH) flows has been the discovery of externally irradiated jets within the confines of HII regions (Reipurth et al. 1998). Similar jets immersed in photoionized medium were identified in the Orion nebula and near the late B stars in NGC 1333 (Bally et al. 2000; Bally \& Reipurth 2001).

\footnotetext{
${ }^{1}$ National Astronomical Observatories, Chinese Academy of Sciences, Beijing 100012, China; ljz@bao.ac.cn

${ }^{2}$ University of Alaska at Anchorage, 3211 Providence Drive, Anchorage, AK 99508
} 
The Rosette Nebula is a spectacular HII region that has been excavated by strong winds from the OB stars at the center of the young open cluster NGC 2244, recently identified as a possible twin cluster based upon near-infrared studies from the 2MASS survey (Li 2005). This emerging young open cluster is found to have a turn-off age of about $1.9 \times 10^{6}$ yrs (Park \& Sung 2002). A set of primarily two jet systems with an externally irradiated origin, HH 889 \& HH 890 (please refer to http://casa.colorado.edu/hhcat/) were discovered in the Rosette Nebula. Note that these two HH jets were formerly called the Rosette HH1 (Li \& Rector, 2004; Li, Chu \& Gruendl 2006) \& HH2 (Li 2003), respectively.

The HH jets discovered in Rosette (Li \& Rector, 2004; Li 2003) and their only rivals in the close vicinity of $\sigma$ Orionis (Reipurth et al. 1998) are found to share many similar properties, which are commensurate with an irradiated origin of the disk-jets systems: (i) all the jet sources are identified in HII regions, bathed in harsh UV ionization from nearby (within a few parsecs) massive OB stars which dominate the excavation of the HII regions. Contrary to conventional jet exciting sources, they show very low extinction along the line of sight and are therefore optically visible, with spectra characteristic of T Tauri stars; (ii) none is detected by IRAS, indicating the lack of circumstellar materials in the form of extended disks and/or envelopes; (iii) all show a normal shock-excited origin of [SII] at the base of the jets, which declines rapidly with distance from the driving source and turns to be $\mathrm{H} \alpha$ dominated, indicating a photoionized nature; (iv) all have a highly asymmetric or even unipolar morphology, indicating perhaps much different jet forming conditions in the launch and collimation regions. As a comparison, jets found in the outskirts of the Orion Nebula show a bipolar but 'C' shaped structure, bending away from the nebula core (Bally et al. 2000).

However, the Rosette $\mathrm{HH}$ jets show additional features not seen in the $\sigma$ Orionis jets. For example, both the HH $889 \& 890$ jets show a high excitation origin, due to perhaps the more intense extreme ultraviolet (EUV) radiation from the exciting OB stars in Rosette, which is 1-2 orders of magnitudes higher than the ionizing fields in orion (Reipurth et al. 1998; Bally et al. 2000). Bally et al. (1998) also found shock structures indicating significant [OIII] emission in the immediate vicinity of the Trapezium stars (within 30" and at a projected distance of $0.06 \mathrm{pc}$ from $\theta$ Ori $\mathrm{C}$ if a distance of $430 \mathrm{pc}$ to the Orion Nebula is adopted), which suggests a shock excited origin of the associated materials and a dependence of the excitation on the intensity of EUV ionization and impacts of fast stellar winds, and hence the distance from the intervening hot OB star. On the other hand, the Rosette jets are not beaming away from any specific OB star or a group of nearby exciting stars in projection. Furthermore, the HH 889 source show in its spectra significant signatures of mass accretion or inflow but no detectable veiling. Successful interpretation of these special features may lead to the final solution of jet formation associated with these and other young stellar 
objects (YSOs).

Li \& Rector (2004) proposed that the discovery of the Rosette jets provides evidence of efficient dissipation of circumstellar disks and envelopes in the vicinity of massive OB stars. This provides indirect evidence for the formation of isolated brown dwarfs (BDs) and free-floating giant planets. UV dissipation of pre-existing protostellar systems in the Rosette by nearby OB stars would prevent these objects from evolving into low-mass stars. Such a mechanism has been shown to be possible and effective by the theoretical studies of Whitworth \& Zinnecker (2004).

It is therefore important to explore the nature of jet formation and disk dissipation of low-mass YSOs in the close vicinity of ionizing sources such as OB stars, as the occurrence of such OB clusters and associations is common in the Galaxy. And our solar system may have formed in such an environment (Looney, Tobin \& Fields 2006). In addition, there has been an unresolved debate on whether there is an evolutionary sequence from classical T Tauri stars (CTTS) to weak lined T Tauri stars (WTTS) as the circumstellar materials around a protostar are gradually exhausted, or they could otherwise be born different in the same regions of active star formation. The latter will require a quick disk dissipation due to external forces after the primordial stage of protostellar collapse and the formation of a protostar. A detailed study of the Rosette jet systems may provide strong test on an abrupt evolution of CTTS to WTTS due to external photoionization of their protostellar disks in massive star forming regions. WTTS formed in this way have indistinguishable evolutionary ages as those of CTTS originated from the same episode of star formation but may indicate substantially different observational properties as will be discussed in this paper.

\section{Observations and data reduction}

Spectroscopic observations of the HH 889 source were carried out with the $2.16 \mathrm{~m}$ telescope of the National Astronomical Observatory of the Chinese Academy of Sciences (NAOC) on Jan. 5, 2005. An OMR (Optomechanics Rsearch Inc.) spectrograph and a Tecktronix 1024 x 1024 CCD were used. A $100 \AA \mathrm{mm}^{-1}$ grating and the 2 " slit resulted in a two-pixel resolution of the spectra of $4.8 \AA$. The spectral resolution of these observations are therefore not significantly better than those employed in the discover of the HH 889 jet (Li \& Rector 2004). However, it is suitable for the faint exciting source of the jet in isolating $\mathrm{H} \alpha$ from the nearby [NII] emission lines.

Two consecutive spectra of the HH 890 source were achieved with the Beijing Faint Object Spectrograph and Camera (BFOSC) and a thinned back-illuminated Orbit $2 \mathrm{k} \times 2 \mathrm{k}$ 
CCD equipped to the NAOC 2.16m telescope on Jan. 4, 2005. Due to the faintness of the HH 890 source with USNO magnitudes of 18.7 in $\mathrm{R}$ and 18.2 in B, respectively, the G4 grating is employed, which gives a two-pixel resolution of $8.3 \AA$.

The spectroscopic data were reduced following standard procedures and packages in IRAF. The CCD reduction included bias and flat-field correction, successful nebular background subtraction, and cosmic rays removal. Wavelength calibration was performed based on helium-argon lamps exposed at both the beginning and the end of the observations every night. Flux calibration of each spectrum was conducted based on observations of at least 2 of the KPNO spectral standards (Massey et al. 1988) per night.

\section{Low-resolution spectra of the jet sources in Rosette}

Fig. 1 presents the spectrum of the $\mathrm{HH} 889$ source around $\mathrm{H} \alpha$. At a resolution of $\sim 4.8 \AA$, the $\mathrm{H} \alpha$ emission is successfully resolved from the nearby [NII] emission lines. This is important as the HH 889 source was found to have complicated profile of $\mathrm{H} \alpha$ (Li \& Rector $2004)$. The forbidden emission lines of $[\mathrm{NII}]$ indicate a line ratio of $\mathrm{I}(\lambda 6583) / \mathrm{I}(\lambda 6548)=3.0$, commensurate with their origin from photoionized medium. The $\mathrm{H} \alpha$ emission, however, shows again an Inverse P-Cygni profile as presented by Li \& Rector (2004) with a lower spectral resolution. A red-displaced absorption feature is clearly resolved, which shows a receding velocity of $\sim 150 \mathrm{~km} \mathrm{~s}^{-1}$ associated with likely mass accretion or inflow. The weak $\mathrm{H} \alpha$ in emission from the source gives an equivalent width of only $1.7 \AA$. This leads to the classification of a WTTS nature of the source based on predominantly the intensity of its $\mathrm{H} \alpha$ emission. This issue will be further elaborated in Section 5.

Here we discuss in detail possible interpretations to the red-displaced absorption associated with $\mathrm{H} \alpha$ : (1) It is due to a continuous accretion or rather inflow of ablated disk materials. However, it is essentially different from conventional IPC profiles as signatures of enhanced accretion onto the central protostar. As noted by Li \& Rector (2004) and convinced by our time series low-resolution spectroscopy, no noticeable veiling in the continuum emission was detected. Could this imply that the accretion flow covers most of the projected surface area of the protostar in the region where most of the absorption is occurring, possibly many stellar radii in front of the star? This, on the other hand, may be closely related to how the jet is sustained in the lack of circumstellar materials as stated below, and poses challenge to traditional knowledge of jet formation and especially mass loading. In this case, EUV ionziation of the relic disk material may have provided an efficient mechanism for mass ejection via a highly deformed star-disk magnetosphere facing the strong radiation field. This could explain why the collimated jet survives its fierce environment. It, however, leads 
to a fast dissipation of the relic disk through both mass ejection and photoevaporation. (2) Alternatively, the absorption line could be due to absorption and obscuration of background nebular emission by the remnant disk materials associated with the jet source (please refer to Fig. 3 of Henney \& O'Dell 1999). Although this does not directly affect emission from the central YSO, it appears as red-displaced absorption in the extracted spectra of the central source after background subtraction, which does not take into account the disk obscuration. However, this feature is specific to the source of $\mathrm{HH} 889$ and was not detected toward neither the HH 890 source ( $\mathrm{Li}$, Chu \& Gruendl 2005, sub.) nor the only parallel jet sources in the immediate surroundings of $\sigma$ Orionis (Reipurth et al. 1998; Andrews et al. 2004).

The spectrum of the HH 890 source is presented in Fig. 2, which illustrates primarily moderate $\mathrm{H} \alpha$, prominent [OIII] emission and shows a late spectral type with a very red continuum in the optical. The equivalent width of $\mathrm{H} \alpha$ varies from 12.2 to $20.5 \AA$ in the two consecutive exposures, this leads to the classification of the jet source as a CTTS based on predominantly its $\mathrm{H} \alpha$ emission. However, its high state of excitation as revealed by its significant [OIII] emission lines strongly suggests a fully ionized origin of the jet and at least the outer layers of the relic disk in association, which will definitely result in a rapid photodissipation of the system.

\section{Photoionization of the jet systems}

Two of the most massive exciting sources of the Rosette Nebula are believed as the dominant sources of ionization and photoevaporation of the jet system associated with $\mathrm{HH}$ 889. One is HD 46223 with a spectral type of $\mathrm{O} 4 \mathrm{~V}(\mathrm{f})$, the hottest star in NGC 2244. It produces Lyman photons at a rate of $10^{49.9} \mathrm{~s}^{-1}$ (Panagia, 1973) and is located 277" or $2.01 \mathrm{pc}$ away from the jet source if a distance of $1.5 \mathrm{kpc}$ to Rosette is adopted (Dorland \& Montmerle 1987). The other is HD 46150, an O5V star at a projected distance of 433" or 3.15 pc from the jet source. It resides at the center of the HII region and produces ionizing photons at a rate of $10^{49.7} \mathrm{~s}^{-1}$ (Panagia, 1973). The combined Lyman continuum emission from these two stars has generated 1-2 orders of magnitude more impact on the disk-jet system associated with HH 889 than that on similar jets discovered in the vicinity of $\sigma$ Orionis (Reipurth et al. 1998) and the Trapezium stars (Bally et al. 2000). The Rosette Nebula is therefore among the most extreme environments in which irradiated jets are found. Though immersed in

photoionized medium, the existence of highly collimated jets strongly suggests the existence of at least a relic disk as a sustained feed to the surviving jet. In the case of the Rosette irradiated jets, a photoevaporating disk with a configuration resembling that of $\mathrm{HH} 527$ in the Orion Nebula as resolved by HST is expected (Bally et al. 2000). This could serve as a 
schematic impression of the appearance of the disk jet system, though in the case of HH 527 , the jet is perhaps oriented at a different direction as respect to the incident UV radiation, is located in the outskirts of the Orion Nebula and has a low excitation state. The physical configuration of the disk-jet systems subject to photoelaboration induced disk-dissipation is believed to be similar.

The HH 890 source is, however, found to be located in a less disruptive UV radiation field, roughly an order of magnitude lower than the HH 889 source faces. That is probably why this jet source still show spectral properties resembling a CTTS, which put constraints on the amount of circumstellar material still in association. However, as stated above, its high ionization status foretells the same fate for this jet system, although it may sustain for a much longer period of time before the remnant disk materials is finally stripped off the central protostar by the UV photoevaporation and dissipation.

\section{Evidence for fast disk dissipation and a young stellar age?}

Being immersed in the fierce UV radiation field of Rosette, the detection of optical jets associated with YSOs indicates either a jet production timescale of as long as 1-2 Myrs, comparable to the evolutionary age of the main cluster NGC 2244, or the YSOs must have a much younger age and the cocoons associated with their protostars had, in some way, been successfully shielded from the strong ionization fields.

Infrared excessive emission is widely taken as an indicator of the existence of circumstellar disks and, hence, youth of the young stars in association. Li (2005) investigated the near infrared properties of the YSOs in the young open cluster NGC 2244 based on the archived 2MASS database. Surprisingly, the jet-driving sources in the Rosette do not show excess emission in the near infrared. Instead, their colors are commensurate with those of WTTS, which have spectral energy distributions indistinguishable from main-sequence dwarves. The HH 890 source, though classified in this paper as a CTTS primarily based on its intensive emission in $\mathrm{H} \alpha$, also shows infrared colors far different from that of conventional CTTS. This suggests that the intense UV radiation exerted by both the external massive stars and the internal protostar has efficiently dissipated the bulk of the disk associated with the YSO. Please refer to their positions on the near infrared color-color and color-magnitude diagrams as presented by Li (2005). This along with the fact that none of the Rosette jet sources and their only rivals found near $\sigma$ Orionis were detected by the IRAS satellite suggests a lack of circumstellar materials as compared to conventional YSOs driving outflows. These are in agreement with the mass estimation of $0.006 \mathrm{M} \odot$, some two orders below the typical value of $\sim 0.1 \mathrm{M} \odot$ of the disks around CTTS, of the relic disk associated with the HH 889 source 
by Li \& Rector (2004). Given the emerging nature of the young open cluster with a turn-off age of 1.9 Myr (Park \& Sung 2002), fast disk dissipation is suggested. Li \& Rector (2004) suggest that this provides indirect observational evidence for the formation of especially isolated BDs and free floating giant planets as discovered in the best studied HII region, the Orion Nebula (Zapatero Osorio et al. 2000), by UV dissipation of unshielded protostellar cores. It can be very important to our understanding of the formation of such sub-stellar objects particularly in regions of massive star formation. Such processes of UV-dissipation could alternatively impose strong effects on the formation and hence the search for extrasolar planets around low-mass stars, the circumstellar disks of which could otherwise be nice sites of terrestrial planet formation. This introduces a viable solution to a long puzzle of how WTTS were formed as a consequence of rapid CTTS evolution and of the rapid dissipation of its circumstellar disk under particular star forming conditions near massive OB stars or in cluster environments. But could the lack of circumstellar materials otherwise indicate an evolved status of the jet sources in Rosette?

The spatial distribution of the two extreme jets in Rosette as respect to the dozens of exciting OB stars of the spectacular HII region is presented in Fig. 3. On which the relic shell structure as indicated by the apparent congregation of excessive emission sources in the near infrared (Li 2005) was superimposed, which suggests the existence of a former working interface layer of the HII region with its ambient molecular clouds. Its projected location of the HH 889 source near the relic arc could well indicate evidence of a triggered origin of its formation in or near the swept-up layer. The HH 890 source has a similar radial distance from the statistical center of NGC 2244 ( $\mathrm{Li} 2005$ ) and may introduce a similar origin. In this scenario, the jet sources have a much younger age than that of the main cluster NGC 2244. Molecular gas and dust associated with the shell may have played an important role in protecting their circumstellar envelopes from the strong UV ionization from the central OB stars. It is therefore reasonable to infer that the jet sources have been directly exposed to the photoionization field just recently.

\section{Conclusions}

We conclude that the Rosette sources driving jets may well represent a transient phase of YSOs evolving rapidly from CTTS to WTTS by fast UV dissipation of their circumstellar disks. This results from abrupt loss of circumstellar materials by fierce photoionization from nearby $\mathrm{OB}$ stars as observed in Rosette, or rather happens in cluster environments due to strong interaction between newly formed cluster members. This, however, poses strong challenge to the traditional knowledge of jet formation, which results from enhanced mass 
accretion and the release of angular momentum of the jet-driving sources. Further detailed studies of the jet systems bathed in photoionized environments may lead to a final solution to the mechanisms of jet formation and collimation. Observations with the Spitzer space telescope are strongly suggested to further investigate the properties of the jet driving sources in the mid-infrared.

We would like to show our great appreciations to an anonymous referee for the help-

ful comments on the paper. This project is supported by the National Natural Science Foundation of China through grant No.10503006.

\section{REFERENCES}

Andrews, S. M., Reipurth, B. \& Ball, J. et al. 2004, ApJ 606, 353

Bally, J., O’Dell, C. R., \& McCaughrean, M. J. 2000, AJ 119, 2919

Bally, J., \& Reipurth, B. 2001, ApJ 546, 299

Dorland, H. \& Montmerle, T., 1987, A\&A 177, 243

Henney, W. J. \& O’Dell, C. R., 1999, AJ 118, 2350

Li, J. Z. 2003, ChJAA Lett. 6, 495

Li, J. Z. 2005, ApJ 625, 242

Li, J. Z. \& Rector, T. A., 2004, ApJ 600, L67

Looney, L. W., Tobin, J. J. \& Fields, B. D., 2006, ApJ 652,1755

Massey, P., Strobel, K., Barnes, J. V., et. al., 1988, ApJ 328, 315

Meaburn, J. \& Walsh, J. R., 1986, MNRAS 220, 745

Panagia, N. 1973, AJ 78, 929

Park, B.-G., \& Sung, H. 2002, AJ 123, 892

Reipurth, B., Bally, J., Fesen, R. A., \& Devine D. 1998, Nature 396, 343

Townsley, L. K., Feigelson, E. D., Montmerlerle, T., et al., 2003, ApJ, in press.

Whitworth, A. P. \& Zinnecker, H., 2004, A\&A 427, 299 
Zapatero Osorio, M. R., Bejar, V. J. S., Martin, E. L., et al., 2000, Science 290, 103

Fig. 1.- Spectrum of the HH 889 source.

Fig. 2.- Spectrum of the HH 890 source.

Fig. 3.- Spatial distribution of the Rosette $\mathrm{HH}$ jets as respect to the ionizing massive OB stars that excavate the HII region. The spectral type of each of the OB stars is also marked on the DSS (Digital Sky Survey) R band image of Rosette. The superposed circles mark the positions of the emerging cluster NGC 2244 (large) and its companion cluster (small) revealed by Li (2005), the enclosed arc structure corresponds to a relic shell harboring triggered star formation in the Rosette Nebula.

This preprint was prepared with the AAS LATEX macros v5.2. 


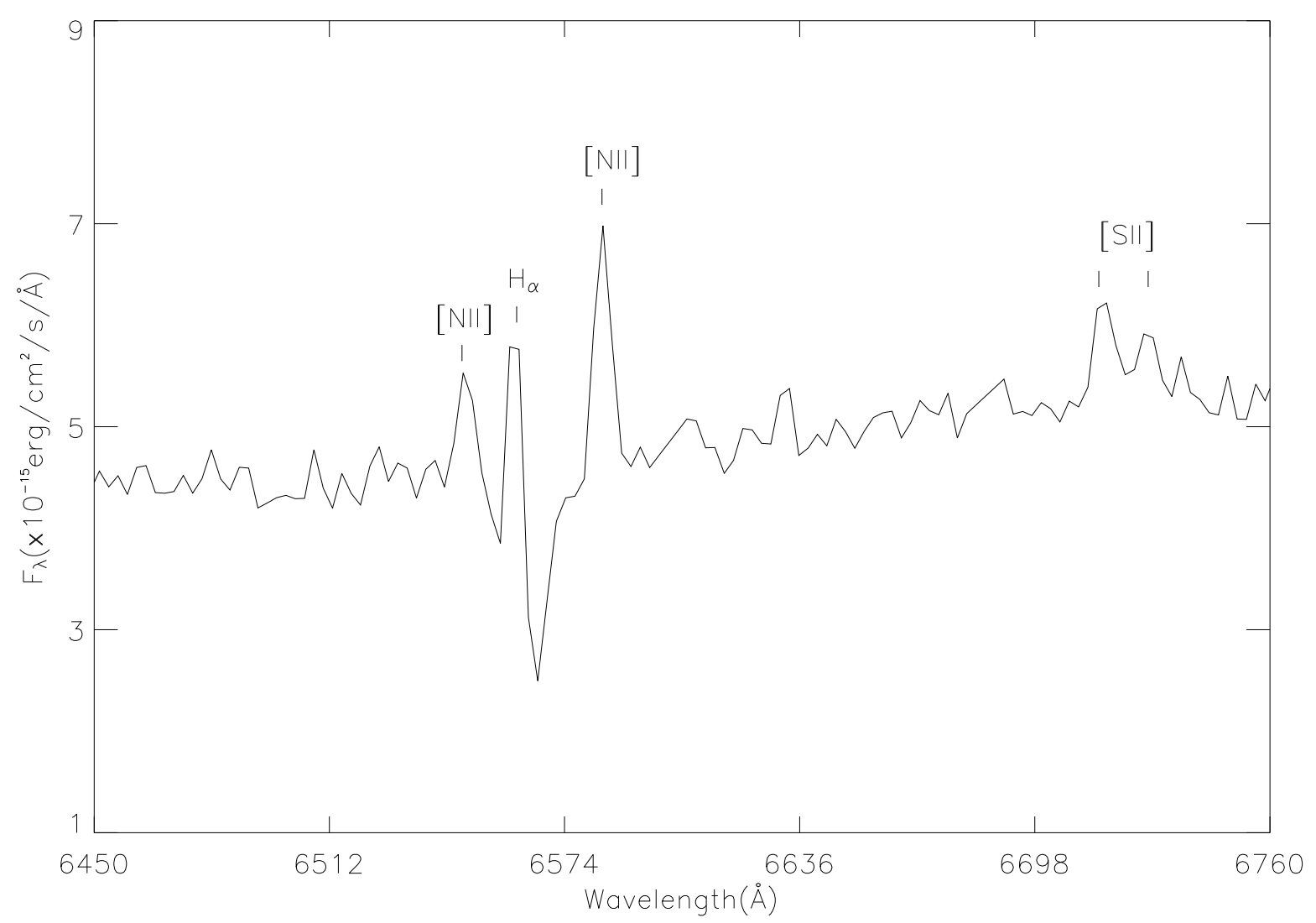

f1.ps 


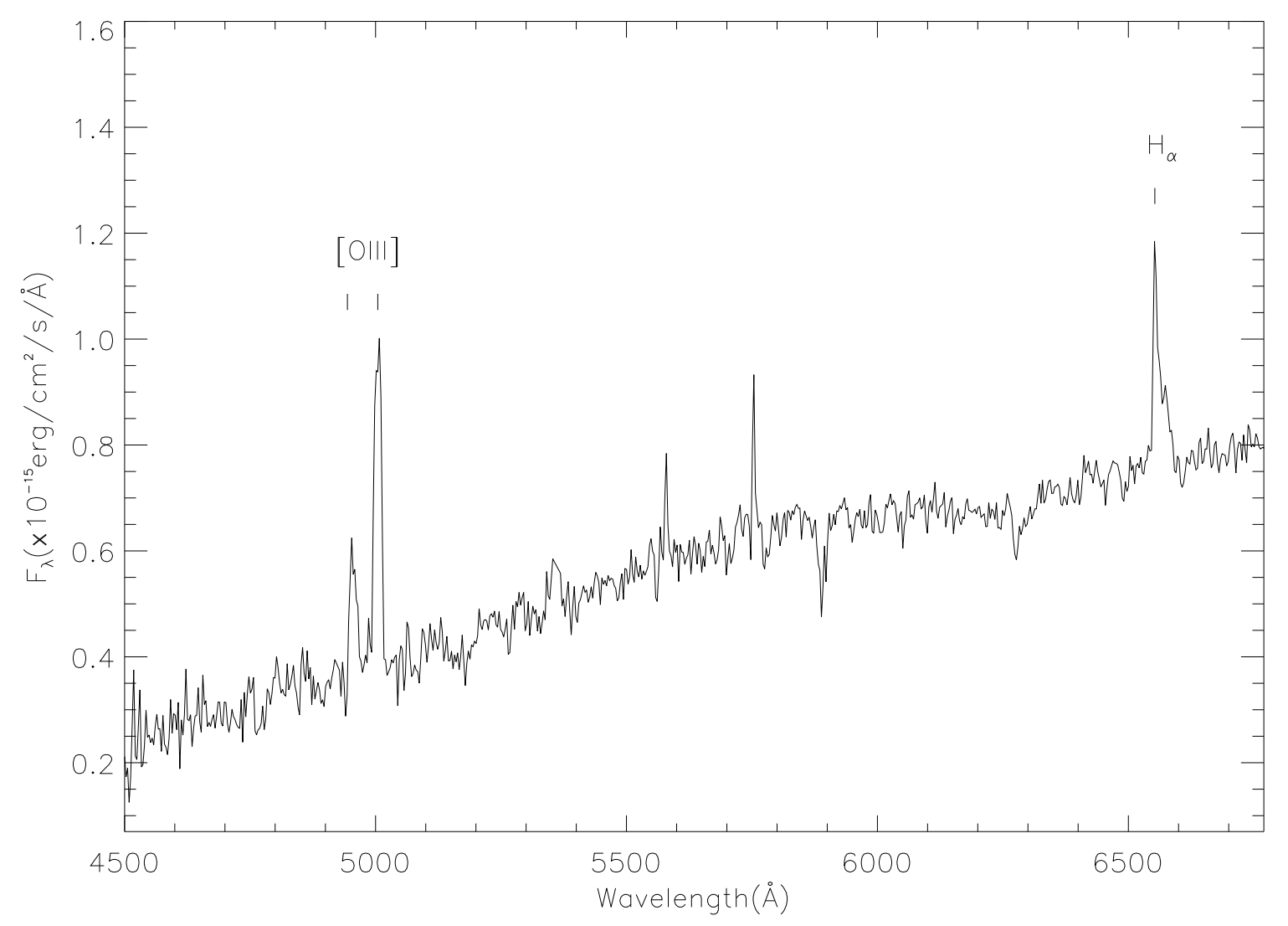

f1.ps 


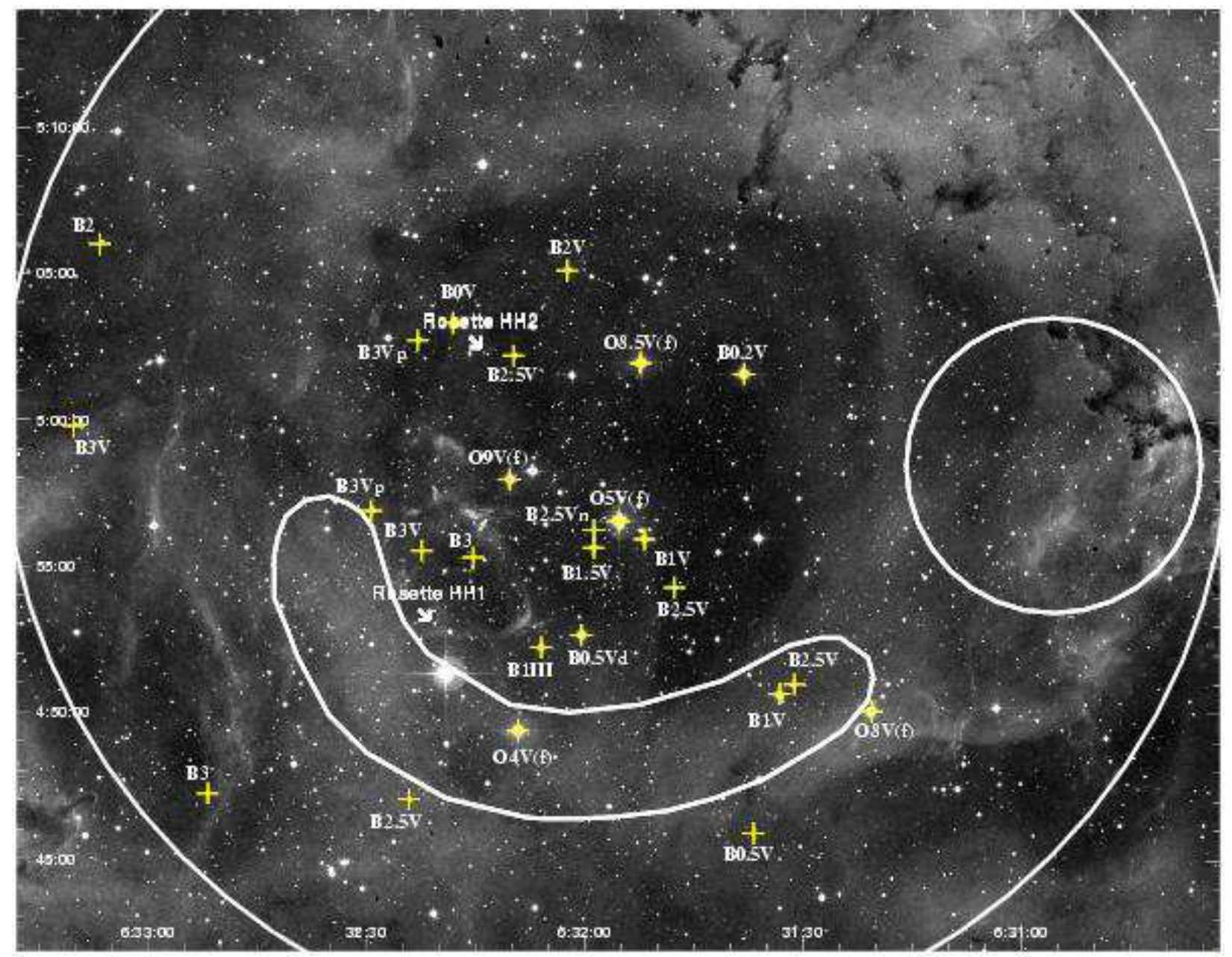

f1.ps 